\title{
LA POSITION DU COMTÉ DE FLANDRE DANS LE ROYAUME Ȧ LA FIN DU XVe SIËCLE
}

\author{
Wim P. BLOCKMANS (Rotterdam)
}

Le roi Louis XI laissait à son successeur un royaume sensiblement agrandi par rapport à l'héritage qu'il avait reçu lui-même. Par l'occupation de la Picardie, de l'Artois, du duché de Bourgogne et de la Franche-Comté, ainsi que par l'incorporation de la Provence et du Roussillon, il avait ajouté $40000 \mathrm{~km}^{2}$ à la France' La royauté continua cette politique dans cette ligne en mettant la main sur la Bretagne ; cette action fut consacrée par le mariage de Charles VIII avec Anne de Bretagne en 1491. Mais l'historiographie n'a pas éclairé la question de savoir si cette extension de l'Etat français visait également l'incorporation du comté de Flandre dépendant, lui aussi, de la souveraineté royale. Si un tel objectif, logique dans le cadre des acquisitions territoriales à cette époque, avait été fixé, pourquoi n'a-t-il alors pas été atteint ?

Il faut constater, en effet, qu'en 1529 François 1 er a renoncé définitivement à « la souveraineté, tout le droit que, en quelque manière il tenoit et avoit, pouvoit tenir et avoir et prétendre ès contez de Flandres et Arthois ». 2 La position de l'Artois différait clairement de celle de la Flandre du fait de l'occupation française et de la constitution en dot de ce pays au profit de la dauphine ${ }^{3}$. En Artois, la tentative royale d'incorporation avait donc pris des formes non équivoques, mais elle a échoué. Le choix de Charles VIII de répudier Marguerite d'Autriche pour se marier avec Anne de Bretagne contribua indéniablement au retour de cette partie de la dot à la maison d'Autriche. Mais qu'en fut-il pour la Flandre, la seule principauté périphérique qui ne fut jamais intégrée à la couronne?

Avant même que la cour de Bourgogne, résidant à Gand, n'apprît de manière certaine la mort de Charles le Téméraire, Louis XI avait lancé ses troupes à la reconquête des territoires que la royauté avait cédés à cette branche

1. P.R. Gaussin, Louis XI. Un roi entre deux mondes. Paris, 1976, 252.

2. Ch. WeIss, Papiers d'Etat du Cardinal de Granvelle. Paris, 1841, t. I er, 464-470.

3. Marguerite d'Autriche fut mariée par le traité d'Arras de 1482 au dauphin Charles, et reçut en dot entre autres terres le comté d'Artois. 
cadette de la maison de Valors ${ }^{4}$ Le complexe territorıal des ducs de Bourgogne s'etait etendu principalement entre 1384 et 1435, c'est-a-dire pendant la phase critıque de la Guerre de Cent Ans Les ducs explottaient la faiblesse temporaire du royaume par un jeu habile d'alliances Les dernieres acquisitions importantes qu'ils purent realiser au detriment de la France, se placent en effet dans le cadre de la paix d'Arras de 1435 Philippe le Bon y fit compenser sa volte-face par la cession obtenue de Charles VII des comtes et seigneuries de Boulogne, Ponthieu, Bar, Mâcon et Auxerre, et celle des villes et châtellenies de la Somme avec clause de rachat ${ }^{5}$

La restauration du pouvoir royal apres 1435 alla de pair avec l'arrêt de l'expansion territoriale bourguignonne dans le royaume, celle-ci s'orientait dorenavant vers les marches de l'Empire au nord et a l'est des Pays-Bas Louis XI a considere comme sa mission primordiale la recuperation des territoires perdus par ses predecesseurs Il y reussit momentanement en 1463 en rachetant, selon les clauses du traite de 1435, les villes et châtellenies de la Somme Succes ephemere pourtant, puisque le duc Charles le Temeraire, en se coalisant avec les princes du royaume, força, en 1465, Lous XI par le traite de Conflans de luı ceder a nouveau la Picardie, en stipulant qu'un rachat eventuel ne pourrait plus se faire avant sa mort ${ }^{6}$

Par le traite de Peronne de 1468 , le rol se vit contraint a faire des concessions supplementaires les proces pendant au Parlement de Paris concernant les limites des comtes d'Artors et de Flandre serarent suspendus pendant huit ans, et le ressort du Parlement en Flandre serait sensiblement restreint ${ }^{7}$

La politıque de Louıs XI contınuait neanmorns a viser les mêmes objectıfs, qu'il poursuivait en harcelant les principautes bourguignonnes limitrophes de la France par des incursions, par de la piraterie et par un blocus economique La destruction des armees de Charles le Temeraire et le desarroi que causa sa mort, offrirent au roı l'occasion rêvee de restaurer le pouvoir royal dans les principautes perıpheriques au nord et a l'est En peu de temps, ses armees occuperent le duche de Bourgogne, la Picardie, une grande partie de l'Artois, et bientôt aussı la Franche-Comte Elles attaquerent Cambra1 et le comte de Hainaut, bien que ces principautes fissent partie de l'Empire, tout comme d'allleurs la Franche-Comte Il n'y pouvalt farre valoir aucun droit Ses

4 Louis XI fut informe de la mort du Temeraire le 9 janvier 1477 GaUssin, Louis XI 270 , Marie de Bourgogne apprit la nouvelle le 24 janvier lettre des duchesses Marguerite $\mathrm{d}$ York et Marie au magistrat de Mons de cette date « ce jourd uy nous ayons oy et entendu que mondit seigneur a este par ses ennemis piteusement occiz et mis a mort " L P GACHARD, « Analectes historiques ", dans Bulletun de la Commisston royale d Historre 2e serie, VII (1855), 6365 Voyez à ce sujet aussı M A ARNOULD, "Les lendemaıns de Nancy dans les "pays de par deça " (janvier avril 1477)", dans W P BLoCKMANS (éd ), Le privlege general et les privtleges provinciaux de Marte de Bourgogne pour les Pays-Bas 1477 (Anciens Pays et Assemblees d Etats, LXXX) Heule 1985,213

5 M R THIELEMANS, Bourgogne et Angleterre Relations polttiques et economiques entre les Pays Bas bourguignons et l Angleterre 1435-1467 Bruxelles 1966, 4956 R VAughaN, Philtp the Good Londres 1970, 98101

6 Weiss, Papiers d Etat I, 912 , Vaughan, Philp the Good 390391

7 K BitTMANN, Ludwig XI und Karl der Kuhne I I Gottingen 1964, 289301 , R VAughaN, Charles the Bold Londres 1973, 5458 
prétentions sur le duché de Bourgogne selon le droit d'apanage étaient aussi peu valides ${ }^{8}$.

Il est clair qu'en 1477 Louis XI ne se soucia pas de la légitimité de ses actes, mais il ne se fia qu'aux rapports de force, son but étant le démantèlement complet de l'Etat bourguignon. Dans les territoires récemment conquis par le Téméraire, il obtint un succès facile : le duché de Gueldre et la principauté ecclésiastique de Liège, secouèrent le joug que Charles leur avait imposé militairement. La pression exercée dans le duché de Luxembourg, où Louis soutint la candidature de Ladislas II Jagellon, roi de Bohême, retarda l'investiture de Marie de Bourgogne et de Maximilien jusqu'en 1480, mais ne put l'empêcher ${ }^{\text {. }}$.

Les tentatives de se rendre maittre des principautés bourguignonnes par un mariage de Marie de Bourgogne avec le dauphin se heurtèrent à une forte résistance de l'entourage proche de la duchesse. Mais aussi les députés des Etats généraux, qui y avaient vu d'abord un moyen adéquat pour parvenir à une paix durable, se montrèrent bientôt réticents. Dès le 28 février 1477 ils firent remarquer au roi que l'invasion militaire des Pays-Bas devait cesser, s'il voulait conclure une alliance matrimoniale ${ }^{10}$. La duplicité de Louis dérouta les députés. La duchesse s'en tint à son alliance avec Maximilien d'Autriche avec qui elle se maria par procuration le 21 avril 1477.

Du coup, Louis fut forcé de donner une base de légitimité à son action dans les principautés bourguignonnes. C'est pourquoi il fit instruire au Parlement de Paris un procès posthume pour lèse-majesté contre Charles le Téméraire. En l'accusant de rébellion et de félonie, il justifiait la saisie des fiefs. L'instruction de ce procès ne fut jamais achevée, les événements prenant un autre cours ${ }^{11}$. Seule, la voie militaire restait en effet ouverte. Après les premiers

8. Gaussin, Louis XI, 271-272.

9. R. PETIT, Le Luxembourg et le recul du pouvoir central après la mort de Charles le Téméraire, dans : Blockmans, Le privilège, 379-384 : le 24 juin 1478, Louis XI transfère à René II de Lorraine ses « droits " sur le Luxembourg auxquels il avait déjà renoncé le 25 novembre 1462.

10. Instructions des Etats généraux à leurs députés près du roi : «Item, et pour ce que telles aliances (de mariage) se doivent traicter par doulceur, et que, se le roi continuoit à entrer et faire marcher plus avant son armée ès pays de par deçà, les subgets de madite demoiselle pourroient prendre tel courage qu'il pourroit retarder et empescher la dite aliance ; suplier au roy que, actendu leurdite inclination (à la paix), il plaise à Sa Magesté faire retraire sadite armée et mectre toutes choses en surséance jusques à ung certain et compétent terme, endedens lequel l'on pourra entendre à ladite aliance. " GACHARD, "Analectes Historiques" ", dans : Bulletin C.R.H., 3e série, $X$ (1869) 274-276 ; R. WELLENS, Les Etats généraux des Pays-Bas des origines à la fin du règne de Philippe le Beau (1464-1506). (Anciens Pays et Assemblées d'Etats, LXIV) Heule 1974, 166-168.

11. GAussin, Louis XI, 275. Nous disposons d'une longue "Justification du droit de Madame Marie de Bourgogne ", rédigée en 1479 par maître Jean d'Auffay, conseiller de la duchesse, en vue d'une procédure au Parlement ou devant des arbitres. L'auteur y réfute systématiquement les arguments avancés par les gens du roi. Les quatre cents articles et plus sont divisés en deux parties, la première traitant du "droit universel possessoire de ma tres redoubtee dame, eschu par le trespas de feu Monsr. le duc Charles », la seconde des droits dans certains pays, principautés et villes en particulier. Le texte est remanié par plusieurs mains, ce qui prouve le soin porté par la cour burgondo-habsbourgeoise à une légitimation suffisante (Archives de l'Etat à Gand, Conseil de Flandre. 34292, folios 14-80).

De cette source, il ressort qu'un nombre considérable de députés d'Artois, de Boulogne et de Saint Pol participèrent aux Etats généraux à Gand en février-mars 1477, et qu'ils y reconnurent Marie de Bourgogne comme la «dame, princesse et contesse naturelle». Le texte cite nommément 8 membres du clergé, 7 nobles présents et 4 autres représentés par lettres, et des délégués de 7 villes (Ibidem, fo $\left.15 v^{0}-16 \mathrm{r}^{\circ}\right)$. 
succès, des résistances se manıfestaıent, tandis que les troupes françaıses avançaıent plus vers le nord. L'opposition de la populatıon d'Arras en fut un premıer symptôme, malgré la défectıon de son capitaıne, Philippe de Crèvecœur, seigneur d'Esquerdes, lieutenant général de la Picardıe ${ }^{12}$.

Les troupes françaıses s'avançaient en Flandre, aux alentours de Lille et de Douai au cours de ma1 et juin 1477. Les dévastations qu'elles causèrent, accrurent toutefois la résistance contre les envahisseurs. Même les villes centrales du comté mirent sur pied des milices pour défendre les frontières ${ }^{13}$.

Le 2 juillet, le roi annonce à la populatıon d'Abbeville qu'il a vaıncu une armée de 10 à 12000 Flamands à Pont d'Espierre ${ }^{14}$ Le 13 août, 1 l informe les magistrats de Lyon de sa victoire sur 8 à 10000 Flamands à La Gorgue, et de la concentration a Nieuwendıjke d'une armee de 28 à 30000 ennemis ${ }^{15}$. Le roi écrıt évidemment à ses bonnes villes pour faire appel à leur soutıen matérıel ; les chiffres cités indiquent suffisamment que la résistance bien que d'une qualité militaire douteuse, fut massive.

Le 27 août, il écrit encore qu'll remet certaines actions afın de pourchasser les Flamands, "quelque part qu'ılz soient " ${ }^{16}$. Le même jour, Maximilien d'Autriche lui proposa la continuation de la paix de 1475 , sous condition de la remıse ımmédiate des terrıtoires occupés. Entre-temps, Maximilien conclut un accord avec les Etats de Flandre pour la formation d'une armée de 5000 hommes pendant 6 mo1s ${ }^{17}$. Louis accepta d'entamer des pourparlers qui menaient à la trève du 18 septembre ${ }^{18}$.

L'invasion fut arrêtée, la Flandre resta intacte sous la maison de Bourgogne-Habsbourg. Et pourtant, le 19 janvier 1477, Louis XI avait bien cla1rement exprımé son ıntentıon de « réunir, remectre et réduyre a la coronne et seigneurie de France les contez de Flandres, de Bourgoingne, Ponthieu, Arthoys et autres terres et seigneuries que nagaires tenort et occupoit feu Charles, de son vivant duc de Bourgoingne $" 19$.

12 Sur ce personnage M HARSGOR, Recherches sur le personnel du Consell du Rou sous Charles VIII et Lous XII 4 vols, Lille-Paris 1980, II, 1077-1116, et pour cet episode 1088 Creveccur s'allid a Louis XI le 2 mars 1477, 1 garda toutes les fonctions qui avalt exercees pour les ducs de Bourgogne

13 Debut mars 1477, les assemblees representatives accordent la mobilisation de 5000 hommes En juin, elles posterent 2000 cavalıers et autant de pietonniers à la frontıere à Pont d'Espierre W P BLockmans, Handelingen van de Leden en van de Staten van Vlaanderen (1477-1506) I, Bruxelles 1973, 17-20 et 26-30

14 J Val SEN, E Charavay, Lettres de Louts XI rol de France 11 vols, Paris 1883-1909, VI, 197-200 Comparez les chiffres sur les effectifs avec ceux, de source comptable, cites dans ld note precedente

15 Ibidem, 216-217 " desconfiz et perdirent ld batalle " Après leur fuite vers le Mont Cassel, ils y furent "desconfiz et tues plus de 4000 »

16 Lettre aux habitants de Harfleur apres avoir rappele ses victorres sur les Flamands, et le marıage (le 18 août) de Marıe de Bourgogne avec Maxımilien d'Autrıche, 1 poursuit " les Flamengs se doivent assembler, nous avons delibere de deldyer aucunes autres entreprises que nous avions pardeça pour les aler trouver avec nostre dicte armee, quelque part qu'ilz soient" Ibudem, 219-220

17 BLOC KMANS, Handelingen I, 36-42

18 Gaussin, Louls XI, 274-275, deux deputes de Gand et deux de Bruges participerent aux pourparlers a Lens BLoC KMANS, Handelingen, 42-43

19 Lettre à Chartres, elle se poursuit amsi avec une double justification " et que paravant luı ont tenues en appainage de ladicte couronne ses predecesseurs, nous solt besoing fare, porter et soubstenır de grans fralz a cause des divisıons et usurpacions que s'efforçoıt faıre 
Le 6 novembre 1477, 1 s'exprime déjà beaucoup plus prudemment : « J'espere en l'aide de Dieu que la chose ura bien en nostre volonté et vouloir pour l'affaire de Flandres $» 20$.

Dans les années suivantes, les hostilités continuent sans victoires éclatantes d'une part ou de l'autre. Après la bataille de Guinegatte du 7 août 1479 , des "faucheurs » français troublent la région de Lille et Douai. Pour sa part, le ro1 pense toujours « parvenır à la réunion de la conté de Flandres", but dans lequel il mobilıse des troupes à la fin de l'année $1480^{21}$.

La mort accidentelle de Marie de Bourgogne le 27 mars 1482 créa une nouvelle situation, avantageuse pour le roi de France à plusieurs égards D'abord, les Etats généraux des Pays-Bas saisirent l'occasion pour forcer Maximılıen à prendre une inıtiatıve de paix, à laquelle ils participèrent activement. Il y consentıt, peut-être parce que les opérations militaires n'offraient pas de perspectives, mais certainement aussi parce qu'il en était réduit à obtenir des Etats sa reconnaissance comme régent au nom de son fils, et pour l'octroi d'une aide ${ }^{2}$. Le résultat de ces négociatıons, menées avec l'accord de Maximilien et en son nom, fut la paix d'Arras du 23 décembre $1482^{23}$. Elle porte les marques du fort désir de paix des Etats généraux ; pour y parvenir, 1ls ont accepté d'importantes concessions. Immédiatement après la mort de Louıs XI, Maxımilien rejeta cette paix.

Un deuxième élément nouveau allait offrir au roı l'occasıon de se mêler des affarres du comté de Flandre. Une grave crise constitutionnelle éclata lorsque les assemblées représentatives refusèrent de reconnaître la régence à laquelle aspirait Maxımilien et que toutes les autres principautés des Pays-Bas lui avalent accordée ${ }^{24}$. Le princıpe juridique en jeu fut la légitımité du testament de Marie de Bourgogne, acte privé en contradiction avec les stipulations du contrat de mariage approuvé par les Etats, quı constituait donc un acte public. Ce dernier, en date du 18 août 1477, avait exclu l'époux survivant de tous droits sur les biens de l'époux décédé. Le testament du 24 mars 1482 ,

a l'encontre de nous et de ladicte coronne ledit Charles de Bourgoingne "Lettres de Louls XI, VI, $115-117$

20 Lettre au seigneur de Curton, ibıdem, X, 394 En janvier 1478, le rol annonce encore au ballı de Vitry son intention d'envoyer une armée " vers les marches de Picardte et de Flandres " Ibrdem, VI, 309

2l Lettres du 30 décembre 1480 aux vılles de Sant-Quentın et Peronne " a ce que puissions plus aisement parvenir a ld reunion de la conte de Flandres, et audit pays, a present a nous rebelles et desobeissans, et donner final aneantissement au fait des guerres et divisions presentes "Ibidem, VIII, 341-345

22 Wellens, Les Etats generaux, 186-194, W P BLOCKMANS, "Autocratie ou polyarchie? La lutte pour le pouvorr politique en Flandre de 1482 a 1492, d'après des documents inedits ", dans Bullettn CR H, CXL (1974), 262-276

23 Texte dans G Doutrepont, O Jodogne, Chroniques de Jean Molinet t I, Bruxelles 1935, 377-406, et J Dumon r, Corps universel diplomatique Vol III, 2, Amsterdam 1726, 100-107

24 BLOCKMANS, "Autocratte ou polyarchie ", 262-276 Je ne partage pas l'opinion de M HARSGOR, Recherches sur le personnel, 1708, selon laquelle Philippe de Cleves aurat soutenu la cause du roı des avril 1477 De même, l'actıvite diplomatıque de Philıpe de Crèvecour comme plenipotentiaire de Lours XI aupres de la ville de Gand, ne peut pas dater du debut 1482, alors que la duchesse Marie etait encore en vie, mats bien d'un an plus tard En execution de la paix d'Arras, Crevecœur fut alors charge de convoyer Marguerite d'Autriche, qu'tl amena à Amboise le 22 juin 1483 Ibıdem, 1093 
dicte par la duchesse mourante, confia a son marı la tutelle et le gouvernement de ses enfants et de tous leurs brens jusqu'a leur majorite ${ }^{25}$

Le conflit politıque fondamental avat pour cause ld volonte de Maximilien de gouverner d'une maniere autocratıque, sans plus tenir compte des coutumes et des privileges de 1477 qui garantıssaient aux grandes villes et aux assemblees d'Etats une forte influence ${ }^{26}$

Au cours des annees 1482 a 1489, Lours XI et les Beaujeu s'applıquerent encore a gagner ce comte de Flandre qui leur avatt echappe en 1477 Selon Commynes, la mort du ro1 ne lu1 permit pas de realiser les desseins sur une Flandre qu'il «tenoit a sa disposition" Seulement, ses droits restaient difficiles a prouver, conclut $M$ Gaussin ${ }^{27}$ Les interventions de la royaute en Flandre eurent, de 1483 a 1493 un caractere exceptionnel, tant par leur frequence que par leur portee Elles se rattacharent a des precedents parfors lointains L'ensemble des relations entre la Flandre et le comte resta controverse jusqu'au jour ou elles furent rompues par la paix de Cambral en 1529 Nous distınguerons quatre domaines dans lesquels l'actıon royale s'est manifestee le territoire, les relations feodales, l'exercice des droits de souverainete et en particulier le ressort jurıdıque

\section{Le territoire}

Bien que l'incorporation a l'Etat frança1s du comte de Flandre se solt averee militairement irrealisable, Louis XI et ses successeurs n'ont pas pour autant ecarte cet objectıf Le tratte d'Arras de 1482 temorgne d'une même logique dans la pensee du rol que son comportement en 1477 , 1 veut obtenır autant de cessions de territorres que possible par la voie matrimoniale C'est en constituant les territoires occupes par la France en dot et heritage de Marguerite d'Autriche qu'il a prevu l'incorporation au domaine des comtes d'Artois et de Bourgogne (Franche-Comte) ainst que celle des terres et seigneurıes de Mâconnaıs, Auxerroıs, Salıns, Bar-sur-Seıne et Noyers, quı devarent revenir a Philippe le Beau et ses heritiers, si le couple restait sans progeniture ${ }^{28}$

Il est interessant de noter que le ror a fait valorr des pretentions sur les villes et châtellenies de Lille, Doua1 et Orchies S1 la dot devait retourner a Philıppe le Beau par defaut d'heritier de Marguerıte, ou parce que le mariage n'etait pas conclu, "le roy serait ent1er audit drott qu'ıl pretend" sur la Flandre gdllicante $\mathrm{S}_{1}$, d'dutre part, le marlage etait rompu de la part du ro1

25 Texte du contrat de mariage Dumon $\mathrm{r}$, Corps untversel diplomatique $\mathrm{III}, 2,910 \mathrm{~L}$ epoux survivant renonce meme aux droits $d$ usage aussi $s$ il $n$ y dvait pas de progeniture, le survivant n aurait aucun droit sur les biens du mort Les Etats de Flandre participerent aux ceremonies du maridge et corroborerent dinst le contrat BLOCKMANS Handelingen, 1,3638 Pour le testament, voyez Y CAzAuX, Marte de Bourgogne Paris 1967326328

26 BLOCKMANS « Brenk of contınuitelt? De Vlaamse privilegien van 1477 in het licht von het stdatsvormingsproces ", dans Le privilege, 115116

27 Gaussin, Louls XI 278

28 Chroniques de Jean Molinet, I, 380381 
avant la majorite de Marguerite, 1 « renonce au rachat » du même territorre ${ }^{29}$. Ce dernier cas se réalisa, comme on sait, en 1491, mais pourtant la question du rachat devait être soulevée à nouveau par Franço1s ler dans sa captivité à Madrid en 1525 ; 1 y renonça défınitıvement par le tratté de Cambraı du 5 août $1529^{30}$.

Quel droit pouvait avoir le roi sur la Flandre gallıcante? Après l'occupation du comté en 1297-1302, Philıppe le Bel avait retenu ce pays en gage de l'exécution du tratté de 1305 et par le tratté de Pontoise de 1312, le comte de Flandre avaıt cédé les trols villes et châtellenıes contre la moitié d'une rente qu'il devalt au roi ${ }^{31}$. Le comte Louis de Male exigea toutefois la restitution de ce territorre et en fit dépendre sa prestation de l'hommage féodal au roi. Il atteignit son but par le contrat de mariage de sa fille unique Marguerite de Male avec Philıppe le Hardi, duc de Bourgogne, mais cet acte du 13 avr1l 1369 stipulait le droit de la couronne à racheter la Flandre gallicante si Philippe le Hardi mourait sans héritıer mâle. La chose fut encore compliquée : a) par un serment secret de Philippe le Hardi à Charles $\mathrm{V}$ de restıtuer le territoire en question après la mort du comte Lours de Male ; b) par des garanties écrites du même Philippe et de son epouse au comte Louis que, non obstant les clauses précédentes, ils n'alıéneront jamais la Flandre gallicante du comté ${ }^{32}$.

Mais sans même pouvoir farre jouer cette dernière garantie, la couronne avait été déchue de son droit par la naissance d'un héritier mâle dès 1371. Si Louis XI rappela la possibilité du rachat, il ne le fit que pour exercer une pression supplémentaire dans les négociations en cours. La même tactique fut d'ailleurs suivie par François ler. Mais il y a plus. Si le roi fait valoir certains

29 Le ler decembre 1481, Louis XI avait deja instruit ses negociateurs qu'lls devatent essayer de faire reconnaître par Maxımilien qu'ıl n'avait aucun droit sur la Flandre gallicante, parce qu'elle avait éte donnee jadis a Philıppe le Hardı a titre viager seulement GaussiN, Louis XI, 276-277 Ld suite de cette matière prouve que les pretentions du roi etaient mal fondees

L'article 6 de la Paix d'Arras prevoit que sı la dot devatt retourner a Philıppe le Beau, le dauphın la garderait « jusques ad ce qu'll soit appointe du droit pretendu par le roy es villes et chastellenres de Lille, Douaı et Orchies, esquelles III villes et chastellenies, se ledit cas de retour ne advenoit, le roy et ses successeurs ne pretendront aucun droit, ma1s en joyront les contes et contesses de Flandres, comme $1 \mathrm{lz}$ ont fatt par cy devant "L'article 28 tratte de la non conclusion du mariage, l'article 84 de la rupture « renonce au rachat des villes et chastellemes de Lille, Douay et Orchies, et consentıra qu'elles demeurent a perpetuite aux contes et contesses de Flandres, sans ce que audit cas soit plus avant enquis ne cognu du drott pretendu par le roy par rachapt ou aultrement " Chromiques de Jean Molinet, 1, 381, 387 et 402-403, DumonT, Corps universel diplomatıque, III, 2, 102, 106

30 Un memorandum date « vers decembre 1525 » quı contıent les reactıons de Françoıs ler aux propositions de Charles-Quint, comporte ces articles (suivant les questions de Bourgogne et de souverannete sur la Flandre et l'Artois) «Propose l'empereur que par ces moyens «l quičtera au roy pour recompense Peronne, Roye, Montdedier et les villes de Somme, ensemble Boulogne et la comte de Guisnes que luy appartient, par ce moyen (seront) estainctes toutes autres querelles d'un coste et de l'aultre - Respond le roy que l'empereur ne quicte rien du sien, car pour Peronne etc, il tient Lille, Douai et Orchies, et quant aux villes de Somme, le roy Loys en rendit argent " Weiss, Papters d'Etat, I, 270-273 Pour la renonciation au « droit de redchat qu'1l (le roi) avoit es seigneuries et villes de Lille, Doudy et Orchies » Ibidem, 464-470

31 F Funck-Brentano, Philppe le Bel en Flandre Paris 1897, H Van Werveke, «Les charges financieres issues du traite d'Athis (1305) ", dans Revue du Nord, XXXII (1950) 81-93 et Miscellanea Mediaevalia Gand 1968, 227-242

32 R Vaughan, Philp the Bold Londres 1962, 5-6 Cette situation est exposee longuement en 52 drticles de la «Justification " par me Jean d'Auffay (Archives de l'Etat a Gand, Conseil de Flandre, 34292, folios 64-78) 
droits - dont il reconnaît qu'ils sont contestables - sur la Flandre gallicante, il renonce de ce fait implicitement à ses droits éventuels sur la partıe flamingante du comté, dépendant en majeure partie également de la couronne.

Pourquoi donc cette distinction? Philippe de Commynes suggère que les députés des Etats généraux, en majorıté néerlandophones, auraient même consenti à la remıse de toutes les parties francophones de l'état bourguignon ${ }^{33}$. On peut douter du bien-fondé de cette opinion pour plusieurs raisons. Il n'y a aucune trace d'une telle attitude qui aurait touché quand même de vastes territoires avec lesquels les Flamands entretenaient des liens commerciaux intensifs. De plus, la Flandre gallicante formait une zone défensive, un cordon sanitaire qui avait protégé le reste du comté contre les incursions françaises des dernıères années. C'est justement pour cette raison que le roi invoqua ses prétendus droits : ayant constaté que l'ensemble du comté ne pouvait être conquis militairement, il préféra obtenir d'abord une base solide, facile à réunir au domaine, quand ce ne serait que par la communauté de langue. Louis XI a évité de répéter l'expérience de Phılippe le Bel, sans toutefois désespérer d'obtenır au moins une partıe du comté, qu'ıl aurait pu ensuite employer pour déstabilıser le reste.

\section{L'hommage féodal}

La paix d'Arras de 1482 stıpulatt que l'héritier de Marie de Bourgogne, Philippe le Beau, l'âge venu, «fera les foy, hommages et devoirs, comme il appartient et que l'on a accoûtumé de faire " ${ }^{34}$. Le 27 janvier 1488, Charles VIII déclare que Maximilien et les trois grandes villes "rendront foi et hommage au Ro1, au nom du duc Philippe, encore en bas-âge " ${ }^{35}$. Le tratté de Senlis de 1493 précisa que Philippe fera hommage à l'âge de vingt ans, en garantie de quoi le maréchal de France Philippe de Crèvecœur gardera provisoirement Hesdin, Aire et Béthune (tandis que le reste de l'Artois était restitué comme faisant partie de la dot de sa sœur répudiée). « Le roi, ses juges et officiers auront la jouissance des ressorts, souveraineté et autres droits qui d'ancienneté ont appartenu aux Rois de France " ${ }^{36}$.

Philippe le Beau fit effectivement hommage au roi pour la Flandre et l'Artois, mais le souci exprimé dans les actes mentionnés s'appuyait sur des expériences bien antérieures ${ }^{37}$. Charles le Téméraire, bien qu'ayant promis en

33 a Et s'ilz luy (le rol) eussent peu farre baller celles de Haynault et de Namur et tous les subgectz de ceste maison qui sont de langue françaıse, ilz l'eussent voulentıers faict pour affoyblir leurdıct seigneur " $\mathrm{Ph}$ de COMmYnes, Memoires, ed J CALMETTE et G Durville Paris 1924-1925, 3 vol, II, 302 Voyez a ce sujet dussi la note 45

34 Artıcle 35 Chroniques de Jean Molinet, I, 389, Dumont, Corps universel diplomantque, III, 2, 103

35 Archives de la ville de Gand, charte no 760

36 Art 5, 6 et 11 Dumont, Corps universel diplomatique, III, 2, 303-305

37 La paix d'Arras de 1435 avalt prive Charles VII de l'hommage feodal de Philippe le Bon ou eventuellement de son successeur, comme vengeance du meurtre de Jean sans Peur En 1461 , Philippe le Bon fit hommage a Lous XI Charles le Temeraire promit à Peronne de faure hommage, sauf si le rol portait atteinte à la Paix d'Arras de 1435, au traite de Conflans de 1465 ou à ses promesses de 1468 Dans ces trols cas, le rol reconnaittrait par le falt même l'independance de tous les fiefs royaux et 1 libererait ainsı le duc Charles et tous ses successeurs de l'hommage, du service, de l'obeissance et de la haute justice au roı De sa part, Louis XI declara dans son manifeste 
1468 dans le traité de Péronne de faire hommage pour ses fiefs royaux, ne le fit jamais. Son attitude envers le roi avait d'ailleurs peu de traits communs avec la foi et la loyauté dues par un vassal. Il voulait au contraire se libérer de toute subordination, même symbolique, envers le roi. Pourtant, pour le roi, ce lien vassalique avait encore une valeur, quand ce ne serait que pour l'assurance que son vassal ne soutiendrait pas des alliances hostiles. Le traité de Conflans de 1465 stipulait en ce sens que le roi cédait les villes de la Somme « sans y retenir aulcune chose, fors les foi et hommage, ressort et souveraineté " ${ }^{38}$.

Les traités officiels n'ont pourtant pas pu arrêter la marche à l'indépendance des fiefs français sous la dynastie de Habsbourg. Dans le traité de Cambrai de 1529, François 1 er renonce définitivement à la souveraineté et « tout le droit que, en quelconque manière il tenoit et avoit, pouvoit tenir et avoir et prétendre ès contez de Flandres et Arthois ».

La réalité du rapport des forces avait rendu illusoire toute réminiscence du statut féodal ${ }^{39}$.

\section{La souveraineté}

Philippe le Bel et ses successeurs sont intervenus régulièrement dans les affaires du comté de Flandre, en vertu de leurs droits de suzerain. La croissance du pouvoir des ducs de Bourgogne et l'affaiblissement du pouvoir royal ont fortement réduit le nombre et la portée de ces interventions. Pendant le conflit entre Philippe le Bon et Gand de 1447 à 1453, la ville chercha l'appui du roi contre son vassal. Le duc lui demanda de ne pas accorder, à la requête des Gantois, des «mandements ou provisions a l'encontre de moy et ou prejudice de ma haulteur et seignourie en icelle ma ville ". Sous la pression du roi il dut néanmoins accepter une trêve. Charles VII n'alla pas plus loin et Philippe le Bon put soumettre la plus grande ville de son état, bien qu'avec retard ${ }^{40}$.

Les crises du pouvoir bourguignon en 1477 et entre 1482 et 1492 offraient de plus grandes possibilités à la royauté. C'est en sa qualité de souverain que Louis XI pouvait prétendre à intervenir en Bourgogne, en Artois et en Flandre "pour préserver les droits de sa filleule ». Charles VIII en fit de même des droits de " nostre très chier et très amé frère et cousin le duc Philippes, conte de Flandres ${ }^{41}$. Le 25 octobre 1484, les régents, le seigneur de Beaujeu et Anne de France promettent leur soutien aux Trois Membres de Flandre « contre

\footnotetext{
d'Amboise du 3 décembre 1470 que Charles, "le soi-disant duc de Bourgogne " était un vassal infidèle, dont il confisquait tous les fiefs royaux et qu'il devait citer devant le Parlement de Paris. Bittmann, Ludwig XI, I, 1, 293 ; W. PaRAVICINI, Karl der Kühne. Göttingen 1976, 32-34. Voyez aussi l'article de M. PARAVICINI (infra p. 183-196).

38. Dumont, Corps universel diplomatique, III, 1, 335-337 ; Weiss, Papiers d'Etat, 1, 9-12 ; Vaughan, Philip the Good, 390-391.

39. WeIss, Papiers d'Etat, 1, 464-470.

40. Lettre de Philippe le Bon à Charles VII du 29 juillet 1451 ; GaCHard, "Analectes historiques », dans : Bulletin C.R.H., 2e série, XII (1859) 362-364. Trêve du 21 juillet 1452 : Archives de la ville de Gani, charte 606; Vaughan, Philip the Good, 323-326.

41. Dans un accord avec Charles VIII datant du 5 février 1485 les Trois Membres de Flandre jurent d'être de bons et loyaux sujets du roi et de la couronne de France, comme de leur souverain seigneur: I.L.A. DIEGERICK, Inventaire analytique et chronologique des chartes et documents appartenant aux archives de la ville d'Ypres. IV, Bruges 1859, 93-94 et 105-106. Voyez aussi note 58.
} 
quiconque prétendrait entreprendre sur la garde et le gouvernement de leur prince ». En février 1488, une instruction de Charles VIII à son ambassadeur à Gand est encore plus claire : il remercie les Flamands parce qu'ils " s'offrent encoires à garder les drois et domaines dudit duc Philippe et au roy les drois de ressors et de souveraineté audit pays de Flandres $"{ }^{42}$.

Comme en 1451-52, les Gantois prirent l'initiative de faire appel à l'assistance du roi dans leur conflit avec Maximilien. Le 18 ma1 1482, Louis XI les remercie de la bonne volonté manifestée à son égard et à celui de la couronne de France ; il rappelle qu'ils recoururent déjà à son père. Il offre ses bons offices aux Trois Etats de Flandre ${ }^{43}$. A la suite de cette lettre, les Trois Membres décıdent à la fin du mois d'envoyer au nom du comté une ambassade auprès du ro1 ; le premier juillet, Louis XI instruit les membres du Parlement de Paris sur les négociations qu'ils devront mener avec les députés flamands ${ }^{44}$. Tout comme en 1477, l'action diplomatıque échoua à cause de la poussée milıtaire simultanée des Français.

Depuis le 28 mars 1482, donc directement après la mort de Marie de Bourgogne, les magistrats de la région frontalière s'inquiétaient des préparatifs militaıres français contre Saint-Omer et Aıre. En mai, les Trois Membres de Flandre mobilisent jusqu'a 20000 hommes pour affronter une invasion éventuelle. Tout de suite après la prise d'Aire par Phılıppe de Crèvecœur le 22 juillet, les troupes flamandes sont dirigées vers Cassel " contre les ennemis qui logeaient aux alentours d'Aire $"{ }^{45}$. Il est clair que l'atmosphère de guerre ne se prêtait pas à la poursuite des avances auprès du roi. La fermeté de toutes les communes flamandes face à l'invasion attendue ne fait nul doute.

La paix d'Arras marque un tournant et ouvre la voie à une collaboration cordiale de la royauté avec les communes flamandes. Dans l'article 36, le roi confirme tous les privilèges, anciens et nouveaux (notamment ceux que Marie de Bourgogne concéda en 1477), généraux et particuliers de la Flandre, de Lille, Douai, Orchies et Saint-Omer ${ }^{46}$. Il pose là un acte législatif de grande portée, en vertu de sa souveraineté. C'est aussi en cette qualité que s'adresse au dauphin l'ambassade des Etats généraux et de Maximilien le 16 janvier $1483^{47}$. Les députés flamands ont profité de leur présence à la cour de France

42 I L A Dlegtrick, Correspondance des magistrats d'Ypres, deputes a Gand et a Bruges pendant les troubles de Flandre sous Maxtmilen Bruges 1853, XIX-XXII

43 VAESEN-ChARAVAY, Lettres de Louls XI, IX, 223

44 Ibidem, IX, 253-254, BLOCKMANS, Handelingen, I, 220

45 BLOCKMANS, Handelingen, 1, 209-211 premiers avertissements de la part de la châtellenie d'Ypres le 28 mars, 214 ravitallement d'Aıre, 216 inquietude dans la châtellenie de Courtra1, "felle nıeumaeren van den Fransoysen» (de fâcheuses nouvelles des Françass) le 30 dvrıl, 219 le $12 \mathrm{md}$, mobilısation dans l'attente d'une invasıon françasse, concentratıon des troupes a Wervik « parce que les Françals s'etalent loges du nombre de 14 ou 15000 hommes a Blangy ", 231-237 emotions a la nouvelle de la prise astucieuse d'Aire, et avance des troupes flamandes

46 Dumont, Corps untversel diplomatıque, III, 2, 103 , Chromiques de Jean Molinet, I, 389

47 «Et pour fare la reverence telle que à filz de roy, heritier apparant de la couronne, et de estre seigneur souverain de ceulx de Flandres, Lılle, Doudy, Saınt-Omer "La delegation est composee des abbes de Saınt-Pierre a Gand, Saint-Bertın a Saint-Omer, Jacques de Goy, haut ballı de Gand, Joris van der Moere et Willem Rijm, premiers echevins de Gand et maitre Jan de Witte, bourgmestre de Bruges Les ambassadeurs sont reçus entre autres pdr certains consellers royaux appartenant a la haute noblesse flamande Jean de la Gruuthuse et Louis de Halewijn, sergneur 
pour solliciter des lettres royaux sur nombre de problèmes particuliers. Le 22 janvier 1483, Lours XI légifère sur le renouvellement des magistrats des trois grandes villes et sur les procédures devant les tribunaux ecclésiastiques dans le comté ; en outre il réforme la procédure du renouvellement du magıstrat de Gand. Dans les trois cas, il entérine entièrement les requêtes des députés des grandes villes ${ }^{48}$. Tel est aussi le cas d'une série d'actes de Louis XI et de Charles VIII, datant de février 1483 à février 1485, et favorisant la draperie de la ville d'Ypres à l'encontre de la draperie rurale, et de deux actes de Charles VIII du 23 mars 1485 en faveur des privilèges commerciaux de Bruges ${ }^{49}$.

Dans le vide politique créé par le refus des organes représentatifs flamands de reconnaître Maximilien comme régent, les rois soutenaient donc puissamment les Trois Membres (Gand, Bruges et Ypres) dans leur ambition d'exercer eux-même la régence. Ils ne se limitaient pas à confirmer des privilèges antérieurs ; ils ont introduit de nouvelles procédures administratives et judiciaires, et ils ont soutenu la politique économique monopoliste des grandes villes. En outre, les rois ont clairement soutenu la cause des Trois Membres dans leur lutte pour le pouvoir politique et économıque vis-à-vis de l'autorité du comte aussi bien qu'envers les petites villes et le plat pays ${ }^{50}$.

En décembre 1483, une délégation du Conseil de Régence de Flandre, composée de Jacques de Savoie, comte de Romont, de Philippe, seigneur de Beveren, de l'abbé de Saint-Pierre à Gand et de Denijs Heyman, pensionnaire de la ville de Gand, devait se rendre chez les régents de France. Les motifs de ce voyage provenaient principalement des soucis qu'avalt ce Conseil de Régence au sujet de sa légitimité depuis que Maximilien qui l'avait installé sous condition, l'avait révoqué en octobre 1483. Les instructions aux ambassadeurs ne contiennent qu'un seul thème : l'argumentation au sujet de la régence en Flandre et les intérêts que pouvaient y avoir les princes de France ${ }^{51}$.

La coïncidence voulut que les Etats généraux du royaume fussent convoqués dans la même période et que les Etats de Flandre y fussent également invités ${ }^{52}$. Il est probable que le Conseil de Régence n’a pas jugé utile

de Plennes L P GAChard, Lettres inedites de Maximilien, duc d'Autriche Bruxelles, 1851, $31-42$ Sur ce dernier personnage HARSGOR, Recherches sur le personnel, 1575-1602

Notons encore l'enthousiasme des ambassadeurs au sujet de l'accueil qui leur fut offert, et ausst de l'attitude du dauphin " longuement 1 l s'est monstre à nous et noz gens, allant et soy pourmenant d'un lieu a aultre, et que les plus de nous ont veu ses jambes et ses cuisses a descouvert » (lettre a Maximilien, datee d'Amboise le 18 janvier 1483)

48 Archives de la ville de Gand, charte $\mathrm{n}^{\circ} 730$, Archives de la ville de Bruges, chartes $\mathrm{n}^{\circ}$ 1189-1190, DItGERICK, Inventaire des archives d'Ypres, IV, 59-60

49 Diegerick, Inventaire, IV, 61-68, 108 , L GILliodTs- Van SEVEREN, Inventaire des chartes des Archives de la ville de Bruges VI, Bruges, 1876, 246-248

50 Notamment les alliances du 25 octobre 1484, conclues entre le seigneur de Beaujeu et Anne de France d'une part et les Trois Membres de l'autre, et du 5 fevrier 1485 entre Charles VIII et les Trois Membres Gilliodts- Van Severen, Inventaire, VI, 241-243 , Diegerick, Inventaire, IV, $105-106$

51 Instructions du 12 décembre 1483 KERVYN de LETTENHOVE, Histoire de Flandre V, Bruxelles 1850, 543-546, BLOCKMANS, « Autocratie ou polyarchie », 281-283

$52 \mathrm{~N}$ BUIST, « Vers les Etats modernes le Tiers-Etat aux Etats generaux de Tours en 1484 » dans R ChARTIER et D RicheT, Representation et vouloir politiques Paris 1982, 20 , la convocatıon fut reçue a Tournaı le 18 novembre 1483 GACHARD, Extraits des registres des consaux de Tournay, 46 Pour la Flandre, nous ne connaissons aucune mention de la participation d'une delegation aux Etats generaux du royaume Le comte ne contribua certainement pas a l'aide 
d'ignorer la convocation, mais qu'il a certainement évité de se laisser entraîner dans les rouages et les problèmes internes $\mathrm{du}$ royaume. La délégation n'avait d'autre mandat que celuı qui concernat la régence ; sur ce point, elle eut l'oreille de l'entourage du jeune roi.

Le 27 décembre 1484, Charles VIII écrit à Maximilien pour l'informer qu'en sa qualité de souverain seigneur, il a conclu un accord de soutien avec Philippe le Beau, comte de Flandre, contre tous ceux qui nuiraient à sa personne ou à son état. Le roi déclare en outre, qu'à la requête de ses sujets flamands, les prétentions de Maximilien sur la " mambournie » et la régence devront être traitées par les pairs du royaume ou le Parlement de Paris. Cette lettre fait suite au commencement des entreprises de Maximilien pour la conquête militaire du comté ${ }^{53}$. En févrıer 1485 , le roi n'hésite pas à accorder son soutien militaire aux Membres contre tous ceux qui entreprendraient quelque chose contre le gouvernement, les privilèges et coutumes de la Flandre. La présence, parmi les témoins de cet acte, d'Antoine, comte de Laroche, grand bâtard de Bourgogne, indique l'influence dans le conseil royal d'un groupe de transfuges bourguignons et flamands qui n'avaient pas coupé tous les liens avec leur milieu originel. Le même Antoine avalt encore joué le rôle de médiateur entre Maxımilien et les Trois Membres en mai-juin 1484, mais sa mission avait échoué ${ }^{54}$.

Toutefois, l'appu1 mılitaire frança1s n'arriva qu'au commencement de mai 1485 , en principe pour « appointer les différends entre le duc d'Autriche et les gens du Conseil (de Régence) du duc Philippe ». Mais, "s'ils (Philippe de Crèvecœur et d'autres consellers) n'y pouvaient parvenir, et que la guerre continuât, il (le ro1) leur avait donné ses ordres pour la défense dudit pays (de Flandre) $"$. La mission des troupes françaises fut tardive, brève et inefficace : le 11 juin, elles s'étaient déjà retirées du comté ${ }^{55}$. La victoire de Maximilien dans le comté fut scellée le 28 juin 1485. Du coup, les interventions royales s'arrêtèrent de manière abrupte. Maximilien avait d'ailleurs fait stipuler dans sa paix que toutes les concessions données par la duchesse Marie ou par le roi de France en contradiction de la paix de Gavere de 1453, devraient être cancellées ${ }^{56}$.

Bien que la victoire de Maximilien en Flandre eût pour conséquence le retrait des troupes françaises et l'arrêt des interventions royales, la guerre continuait en Artois. Philıppe de Crèvecœur, maréchal de France et lieute-

53 P Pelicier, Lettres de Charles VIII, roi de France I, Paris 1898, 50-56, Blockmans, "Autocratie ou polyarchie", 289

54 Ce groupe d'interêts flamands et artesiens comportait en outre Lous de Halewijn, Jean de la Gruuthuse, et surtout Philıppe de Crevecoeur, seigneur d'Esquerdes HARSGOR, Recherches sur le personnel, 1077-1116, 1576-1602, 2356-2367, sur ld mediation d'Antoine de Bourgogne BLOCKMANS, "Autocratie ou polyarchie ", 284-287 En decembre 1483, le Consell de regence de Flandre avalt fait appel à lui pour "garder " le droit de Philippe le Beau KERvYN de LETTEN HOVe, Histoire de Flandre V, 543-546

55 Pflicier, Lettres de Charles VIII, I, 77-78, GaChard, Extraits des registres, 50-56 le 7 maı 1485, l'arrıvee des troupes françatses est discutee par les magıstrats de Tournal, tout comme leur retour le 11 juın Le magistrat essayd de garder la neutralité dans le conflit Le recti de Jean Molinet precise que Crevecœur commanda 500 lances et 4000 pietons, qu'il diriged le 11 mai vers Courtrai Le 11 juin, 1 quittd Gand a la requête du magistrat Ses troupes, que Molınet estıme mantenant a 500 lances et 6 a 7000 pietons, auraient seme la terreur parmi la population Le même jour, ll seratt deja arnve pres de Tournat Chrontques, I, 446-447, et 455-456

56 Blockmans, "Autocratie ou polydrchie ", 290-291, DIEGERICK, Inventaire, IV, 112-114 
nant-général en Arto1s et Picardie, put reprendre Saint-Omer et Thérouanne. Les deux parties accusaient chacune l'autre de rompre la paix d'Arras. Maximilien expliquait à ses sujets que, depuis la mort de Louis XI, les régents du royaume l'attaquaient sur tous les fronts dans le but de le chasser des pays qui lui restatent. Selon le roi des Romains, les Français avaient même trouvé moyen de provoquer une rébellion à Gand et puis dans la Flandre entière ${ }^{57}$. La ville de Gand fut en effet la première à rejeter l'autorité de Maximilien en novembre 1487 , et à se rallier à la royauté ; les autres grandes villes ne suivirent qu'après de fortes réticences, le 7 mars $1488^{58}$. Charles VIII prit sous sa protection tous les Flamands qui réitérèrent leur fidélité à la paix d'Arras ; il leur garantit le libre commerce dans tout le royaume et ordonna à Philippe de Crèvecœur de prendre en mains, avec les Trois Membres, le rétablissement de l'ordre et de la paix. Il est intéressant de noter que le roi souligne qu'il n'a d'autres prétentions que de "deffendre... les subgetz du royaulme " ${ }^{59}$.

L'influence, à la cour de France, de Philippe de Crèvecœur surtout, mais aussi d'autres transfuges flamands et bourguignons, comme le Grand Bâtard Antoine et Louis de Halewijn, seigneur de Piennes, favorisa sans doute l'ingérence croissante de la couronne dans le comté ${ }^{60}$. Le 17 janvier 1488 , Charles VIII concède à la ville de Gand, à sa requête, le droit de battre monnaie d'or et d'argent au nom du comte Philippe et de nommer des fonctionnaires comtaux; il confirme tous les privilèges de la ville, dont Maximilien avait cancellé certains en $1485^{\circ}$. Une série d'interventions confirme les actes posés par les révoltés et leur assure son soutien. Le 22 octobre 1488, Charles VIII met à leur disposition les biens de leurs adversaires ${ }^{62}$. De nombreuses délégations de la part des Trois Membres de Flandre se succèdent à la cour en 1488 et 1489 , pour demander avec insistance un soutien réel ${ }^{63}$.

Il est étonnant que, même après les paix de Francfort et de Tours en 1489 , le roi ait continué de procurer son appui matériel et politique à la ville de Gand et à Philippe de Clèves ${ }^{64}$.

57 GaChaRd, Lettres de Maximilien, 61-65 (lettre de Maximilien au magistrat d'Ypres du 11 février 1487), 68-72 (lettre de Gand a Mons du 10 novembre 1487), 113 (lettre de Maxımilien dux Etats de Hainaut du 21 juillet 1488)

58 Diegcrick, Correspondance des magistrats d'Ypres, XIX-XXII, DiEgerick, Inventaire, IV, 134-136, Archives de la yille de Gand, Chartes n ${ }^{\text {os }} 757$ (20 janvier 1488), 760 (27 janvier) et 761 (6 mars), Blockmans, «Autocratie ou polyarchıe », 293-298, Blockmans, Handelingen, I, 445-446 delegation auprès du ro1

59 « Ne pretend n1 ne veult pretendre drott, querelle et action aucune, ne lever prouffit ou denier, mais se veult emploier a le garder au prouffit dudit duc Phelippe son frere, et deffendre les subgetz desdits pays de toutes forces et oppressions comme les subgetz du royaulme " DIEGF RICK, Correspondance XXII

60 HARSGOR, Recherches sur le personnel, 1094-1096, 1588-1594 et 2362-2364 $275-280$

61 Archives de la ville de Gand, chartes $\mathrm{n}^{\text {os }} 754-756$, Pelicier, Lettres de Charles VIII, I

62 Ibudem, 767 , Diegerick, Inventatre, IV, 152 , Pelicier, Lettres de Charles VIII, I, 307, $343-345$, II $, 145,207,251-253,278,284,317-319,386-387$

63 Blockmans, Handelingen, I, 458, 466, 492-493, 507-510, GACHARD, « Analectes historıques ", dans Bulletin $C R H, 2^{\text {e }}$ serie, XII (1859), 395-398

64 Le 12 octobre 1491, Charles VIII fait livrer a L'Ecluse et a Gand du blé et du vin, le 19 octobre, $\mathrm{t}$ confirme tous leurs dctes poses en son nom et 1 reitere le droit de battre monnaie Archives de la ville de Gand, chartes $\mathrm{n}^{\circ}$ 779-781, Blockmans, Handelingen, I, 529 
Le 24 avril 1488, Philippe de Crèvecœur informa le magistrat de la ville de Bruges que «toutes choses (étaient) prestes » pour mettre les adversaires du roi « à la raison ». Au sein du conseil royal, la chose n'est toutefois pas si évidente. Début juillet 1488 , les actions des troupes françaises ont quelque succès; l'espoir que pouvaient avoir les Bretons d'être secourus par des troupes allemandes, est déçu ${ }^{65}$. Crèvecœur insiste sur un effort prioritaire en Flandre afin d'y stabiliser la situation pour longtemps. Il calcule que le gros de l'armée française pourrait être prêt plutôt en Flandre qu'en Bretagne. A son avis, un succès en Flandre en entraînerait un autre en Bretagne, mais pas inversement. La majorité du conseil donnạ pourtant la priorité à la conquête du duché, les deux actions étant mises en concurrence ${ }^{66}$. Le 9 août, le roi, tour en se félicitant de l'appui que les Flamands ont procuré aux capitaines français, déclare qu'il ne peut pas provisoirement envoyer les «gens d'armes et arbalestriers " requis. Ils viendront dès que "cest affaire de Bretaingne (sera) parachevé, qui sera de brief $"{ }^{67}$. Vers le 18 août, les troupes françaises tenaient Dunkerque et Bergues, deux villes dans le sud-ouest du comté, mais leur contrôle se limitait à cette zone-là.

Dans cette situation, on comprend que Crèvecœur ait proposé aux villes et châtellenies de Lille, Douai et Orchies d'entamer des négociations de paix séparées. La Flandre gallicante se montra fidèle à Maximilien et lui demanda comment réagir ; évidemment, il repoussa toute paix partielle qui tendrait à affaiblir son emprise sur une région visée par la couronne depuis longtemps ${ }^{68}$. La Flandre flamingante était divisée entre des villes comme Dixmude, Nieuport et Furnes, restées sous la domination de Maximilien, et celles plus au sud-ouest, qui soutenaient la cause des Trois Membres et de la France. Celles-ci contribuèrent substantiellement à l'entretien des troupes françaises, tout comme les autres devaient payer des garnisons allemandes ${ }^{69}$. La présence en Flandre de conseillers royaux comme Louis de Halewijn, seigneur de Piennes, et Jean de Gruuthuse, facilita sans doute la collaboration entre Flamands et Français. Mais, malgré l'insistance des Trois Membres, la majorité du Conseil royal ne voulut pas envoyer de nouvelles forces en Flandre avant la fin d'avril $1489^{70}$. Elles arrivèrent trop tard pour éviter un carnage parmi les milices flamandes près de Dixmude en mai 1489. Malgré leur nombre très important, estimé par Jean Molinet à 16000 à 18000 hommes et leurs « gros engiens dont ilz avoyent grant largesse », elles durent lever le siège de Nieuport

65. Lettre de Charles VIII à Louis de la Trémoille du 8 juillet 1488 : « vous verrez comment les affaires de Flandres se portent et comment nous y sommes servy ; il ne fault jà que les Bretons s'actendent à avoir secours des Allemans ». PELICIER, Lettres de Charles VIII, II, 145. Voyez aussi, pour le bon accueil de Louis de Halewijn, seigneur de Piennes, à Ypres, et pour la collaboration d'Ypres avec Crèvecour : GaCharD, Lettres de Maximilien, 103-109.

66. Lettre de Crèvecœur à Charles VIII du 17 juillet : " Sire par le train que lesdicts Bretons tiennent, il peut sembler qu'ils ne quierent que gaigner temps pour veoir l'issue de l'affaire de Flandres, scachans que cellui de deça emportera le fait de Bretaigne, et non pas cellui de Bretaigne l'affaire de Flandres ", citée par HARSGOR, Recherches sur le personnel, 1094-1096; aussi 1588.

67. GaChard, Lettres de Maximilien, 145.

68. Ibidem, 183-186 : réponse de Maximilien aux gouverneur et Etats, datant d'environ décembre 1488. Le ler février 1489 Crèvecœur fut envoyé à Tournai pour y négocier éventuellement avec Maximilien: GACHARD, Extraits des registres de Tournay, 75.

69. Diegerick, Inventaire, IV, 154-157; BlockmaNS, Handelingen, I, 498-502.

70. PELICIER, Lettres de Charles VIII, II, 317.319: lettre aux Trois Membres du 18 avril 1489 ; DIEGERICK, Inventaire, IV, 160. 
au bout de neuf jours le 28 juin. L'armée française ne montrait plus le même éclat, et bientôt la paix de Francfort lui enleva sa mission ${ }^{7 !}$.

\section{Le ressort juridique}

L'exercice de la justice est une éminente prérogative souveraine. Le Parlement de Paris était l'institution qui exerçait par excellence cette fonction. Pendant la seconde moitié du XVe et les premières décennies du XVIe siècle, la compétence du Parlement a souvent été l'enjeu de la lutte entre les rois et les comtes de Flandre. En 1445, les Quatre Membres c.à.d. les trois grandes villes et le district rural du Franc de Bruges, en leur qualité de cours supérieures, obtinrent du roi, moyennant de grosses sommes d'argent, un sursis de neuf ans d'appel contre leurs sentences ${ }^{72}$. Cette situation fut prolongée jusqu'en 1463. Alors, Louis XI a ostensiblement cherché la confrontation : de 1464 à 1468 , la moyenne annuelle d'arrêts et jugés concernant la Flandre monte de 10 à 15, et un nombre appréciable concerne des appels contre des sentences des échevins des Quatre Membres (voir tableau I ci-après). Plusieurs arrêts contrarient ostensiblement les cours supérieures du duc de Bourgogne, à savoir son Grand Conseil et sa Chambre du Conseil en Flandre ${ }^{73}$.

On comprend dès lors mieux l'actıon de Charles le Téméraire à Péronne en 1468 : non seulement il fait renouveler par Louis XI la franchise d'appel des "quatre lois principales de Flandre", mais il fait aussi interdire tous les appels omisso medio. Un article est en outre consacré à la Flandre gallicante : selon ce texte, les villes et châtellenies de Lille, Douai et Orchies avaient été jointes au comté lors du mariage de Philippe le Hardi « pour les tenir par ledit comte en un seul fief avec ledit comté ». Cela implique qu'également les appels de cette partie du comté ne pourront être reçus qu'après avoir été relevés devant le gouverneur ou le Conseil de Flandre ${ }^{74}$. On remarque ici que la politique visant à détacher la Flandre gallicante du reste du comté fut consciemment menée aussi par la voie judiciaire.

Dans la pratique, les arrêts et jugés du Parlement sur appels flamands s'arrêtèrent complètement de février 1471 à 1483. La paix d'Arras avait en effet à nouveau modifié la procédure : les appels de la Flandre gallicante devaient aller en ressort direct au Parlement de Paris, et ceux provenant de la Flandre flamingante être convertis en réformation ${ }^{75}$. De 1484 à 1489 on note une légère reprise de l'activité judiciaire, qui s'arrête toutefois à nouveau totalement de 1490 à 1494. La paix de Montıls-lès-Tours avait pourtant seulement confirmé les stipulations de 1482. Contrairement aux mutations antérieures de la

71 Chrontques de Jean Molmet, II, 133-141

72 W P BLockmaNS, De volksvertegenwoordiging in Vlaanderen (1384-1506) Bruxelles 1978, 533 Le 16 févrıer 1447, Phılıppe le Bon dut rappeler le roı à la surseance, pour faire arrêter un appel contre les échevins de Bruges Gachard, "Analectes historiques ", dans Bulletın $C R H$ 2e serie, XII (1859) 359-362

73 Nous avons base nos conclusions sur l'admirable édition de M R C Van CAEnEgem, Les arrêts et juges du Parlement de Parts sur appels flamands, conserves dans les registres du Parlement 2 vols parus (1320-1521), Bruxelles 1966-1977

Nous ne rendons donc pas compte des procès mentionnés dans la série des Accords, nı dans celle des Plaldoiries

74 Dumont, Corps universel diplomatique, III, 1, 399

75 Ibıdem, III, 2, 103 , Chrontques de Jean Molınet, I, 389 
TABleau I

Fréquence des arrêts du Parlement de Paris sur appels flamands

\begin{tabular}{|c|c|c|c|}
\hline Période & $\begin{array}{c}\text { Nombres d'arrêts et } \\
\text { jugés enregistrés }\end{array}$ & $\begin{array}{c}\text { Moyenne } \\
\text { annuelle }\end{array}$ & $\begin{array}{c}\text { Nombre d'appels } \\
\text { contre les quatre } \\
\text { lois principales }\end{array}$ \\
\hline $1446-1463$ & 172 & 10 & 2 \\
$1464-1468$ & 75 & 15 & 9 \\
$1469-1471$ & 18 & 9 & 0 \\
$1472-1483$ & 0 & 0 & 0 \\
$1484-1489$ & 22 & $32 / 3$ & 3 \\
$1490-1494$ & 0 & 0 & 0 \\
$1495-1521$ & 107 & 4 & 16 \\
\hline
\end{tabular}

pratique judiciaire, il n'y avait donc aucune stipulation normative nouvelle en 1490. On peut voir dans ce nouvel arrêt une manœuvre politique du roi dans le but de rallier encore les Flamands en les contrariant le moins possible par l'exercice de ses prérogatives juridiques. Cette attitude coïncide avec d'autres actes royaux en faveur de la ville de Gand en cette période.

Après la restauration par le traité de Senlis en mai 1493 de tous les ressorts dont jouissaient anciennement les rois, ses juges et officiers, l'activité judiciaire du Parlement en Flandre reprend ${ }^{76}$. On constate un nombre élevé d'appels contre des sentences des " quatre lois principales", avec de nombreux cas où les échevins encourent une amende pour mauvais jugement. Après l'installation du Grand Conseil de Malines, en 1504, le Parlement s'engage directement dans les conflits de compétence.

Les stipulations explicites du traité d'Arras concernant le statut particulier de la Flandre gallicante méritent une attention spéciale. Le tableau II montre

TABleau II

Fréquence des arrêts du Parlement de Paris sur appels provenant de la Flandre gallicante

\begin{tabular}{|c|c|c|c|c|c|c|c|}
\hline \multirow[b]{2}{*}{ Période } & \multicolumn{3}{|c|}{ Nombre d'arrêts } & \multicolumn{4}{|c|}{ Instances appelées } \\
\hline & Total & Fl. gallic. & $\%$ & Echevins & $\begin{array}{c}\text { Gouver- } \\
\text { neur }\end{array}$ & $\begin{array}{l}\text { Conseil } \\
\text { de Flandre }\end{array}$ & Autres \\
\hline $1446-1463$ & 172 & 57 & 33 & 9 & 35 & 5 & 8 \\
\hline $1464-1468$ & 75 & 23 & 31 & 8 & 9 & 3 & 3 \\
\hline $1469-1471$ & 18 & 7 & 39 & - & 6 & - & 1 \\
\hline $1472-1483$ & $一$ & - & & & & & \\
\hline $1484-1489$ & 22 & 5 & 23 & - & 1 & 4 & - \\
\hline $1490-1494$ & - & - & & & & & \\
\hline \multirow[t]{2}{*}{$1495-1521$} & 107 & 17 & 16 & $\sim$ & - & 17 & - \\
\hline & 394 & 109 & 28 & & & & \\
\hline
\end{tabular}

76. Ibidem, III, 2, 305: « Le roy, ses juges et ses officiers arront la joyssance des ressors, souveraineté et d'aultres droits qui d'anchiennetté ont appartenu aux Rois de France et dont,les juges et officiers royaux ont accoustumé cognoistre et joyr. " Chroniques de Jean Molinet, II, $357-358$. 
que les arrêts sur appels provenant de la Flandre gallicante constituaient, de 1446 à 1471, un tiers de l'ensemble des appels flamands. Compte tenu du fait que Lille, Douai et Orchies ne comportaient avec leurs châtellenies qu'un dixième de la population du comté, le Parlement enregistrait donc par habitant trois fois plus d'arrêts dans la partie francophone du comté que dans son ensemble. La langue et la distance pourraient être invoquées comme explications. Ces facteurs n'expliquent pourtant pas la forte baisse proportionnelle des arrêts de la Flandre gallicante à partir de 1482 (baisse de $33 \%$ avant 1471 à $23 \%$ et $16 \%$ depuis 1484 ). La politique royale qui, jusqu'en 1529 , n'avait pas encore délaissé ses espoirs de regagner cette région, pourrait avoir mené à une plus grande souplesse de la part du Parlement ${ }^{77}$.

On ne peut que conclure de ces données que les rois ont utilisé leur ressort juridique sur la Flandre comme une arme politique, servant leur but de détacher la Flandre gallicante, d'exciter ou d'amadouer les sujets flamands selon les besoins de leur cause. Très active pendant les périodes où l'intervention politique était absente, la juridiction royale se mitigeait sensiblement dans son action, au point de devenir inexistante, pendant les périodes d'options politiques ouvertes.

\section{Conclusions}

Les tentatives des rois de France de réintégrer la Flandre dans le domaine, ne se limitent pas aux efforts matrimoniaux et militaires très poussés de 1477 . A partir de 1482, la minorité du comte Philippe le Beau leur offrit de nouvelles occasions. Ils ont soutenu les grandes villes dans leur résistance contre Maximilien avec des moyens politiques, juridiques, judiciaires, militaires et économiques. Cette politique n'atteignit pas son but maximal : la récupération de la Flandre entière, ni même de la Flandre gallicante seulement. Les raisons en sont diverses.

1. Après un siècle de régime bourguignon, une cohésion dynastique avait pris force en Artois, en Flandre, en Brabant et même en Hainaut (bien que ce comté n'ait été conquis que depuis un demi-siècle). La Flandre et l'Artois étaient plus fortement liés économiquement et mentalement aux autres principautés des Pays-Bas bourguignons qu'à la France. L'action des Etats généraux en témoigne amplement.

2. Les actions militaires des Français ne pouvaient que créer un sentiment d'aversion de la part des sujets : ne citons que le sac d'Arras en 1477 et les dévastations continuelles du plat-pays. Les milices flamandes sont d'ailleurs sorties pour défendre le pays contre les envahisseurs français en 1477. Les troupes françaises placées en garnisons au sud-ouest du comté en 1488 et 1489 causaient des charges exorbitantes pour les habitants. Répétons la citation de

77. La population totale du comté peut être estimée vers 1470 à environ 734000 habitants, celle de la Flandre gallicante à 73400 : W. Prevenier, «La démographie des villes du comté de Flandre aux XIVe et XVe siècles » dans : Revue du Nord, LXV (1983) 255-275.

Avant 1471, un appel fut enregistré par 844 habitants en Flandre gallicante contre un par 2770 habitants dans l'ensemble du comté (proportion : 1 à 3,28 ). 
Philippe de Commynes selon qui Jean de Daillon, seigneur de Lude, espérait devenir gouverneur de Flandre et s'y couvrir d'or ${ }^{78}$.

3. Le roi soutenait les trois grandes villes, même dans leur exploitation du plat-pays. Ainsi, peu de sympathie naquit dans le reste du comté pour la cause française, dont on n'observait que les aspects négatifs. De plus, les régimes révolutionnaires dans les grandes villes étaient extrêmement instables, chancelant dès que des rumeurs populaires, des détériorations du niveau de vie ou des débacles militaires tournaient à leur désavantage. Les alliés du roi se tournaient vers lui par faiblesse, et ne pouvaient ni ne voulaient défendre sa cause à fond.

4. Les moyens militaires français investis dans la lutte pour le pouvoir furent par moment importants. Leur présence fut toutefois irrégulière et tardive, laissant ainsi des chances à l'ennemi. L'appui que pouvaient leur procurer les milices communales relevaient plutôt du folklore. De son côté, Maximilien mit du temps à mettre sur pied des armées pour soumettre la Flandre, mais il finit par disposer d'une force importante menée par des stratèges supérieurs comme Albert de Saxe.

5. Si Louis XI voulait à tout prix détruire la puissance bourguignonne, en partie en raison de rancunes personnelles, les intérêts de Charles VIII et de ses régents étaient plus divers. Dans le Conseil royal, des factions défendaient des intérêts contradictoires ; celle de Crèvecœur, défendant la priorité " des affaires de Flandre » fut évincée par le groupe autour de Louis de la Trémoille qui fixait la conquête de la Bretagne comme le but principal. D'autres intérêts internationaux pesaient plus lourd enfin, ce qui amena Charles VIII à faire la paix avec Maximilien dès juillet 1489. En renvoyant Marguerite d'Autriche avec sa riche dot comprenant l'Artois, la Franche-Comté, Auxerre, Bar, Noyers et Charolais, afin d'épouser Anne de Bretagne, Charles VIII montra que la conquête de la Flandre n'était plus pour lui un but primordial.

6. Sans doute, la majorité du Conseil royal s'est rendu compte des obstacles énormes à l'incorporation de la Flandre. A la différence de la Bretagne, le pays était densément peuplé, hautement urbanisé et très développé sur les plans économique, social et culturel.

Les privilèges que concédaient Louis XI et Charles VIII aux grandes villes flamandes auraient été inconcevables dans le cadre de leur propre Etat.

Ces communes aspirant et habituées aux libertés auraient d'ailleurs supporté encore moins l'autorité royale qu'elles ne supportaient celle de leurs comtes. Cette contradiction profonde entre les alliés de circonstance resta couverte mais serait apparue très vite après une élimination hypothétique de l'ennemi commun qu'était Maximilien.

Il aurait donc toujours été difficile de maîtriser une telle concentration de pouvoirs à la périphérie du royaume. Philippe le Bel en avait déjà fait l'expérience. Cela n'empêcha pas Louis XI et certains conseillers de Charles

78. Cité par M. HARSGOR, « Maitres d’un royaume ; le groupe dirigeant français à la fin du XVe siècle » (infra, p. 140). 
VIII de la renouveler sérieusement. Seule la Flandre gallicante et le sud-ouest furent détachés du comté sous Louis XIV : à peu près les territoires visés entre 1477 et 1529. Ces continuités suggèrent l'existence de mécanismes plus profonds dans la formation et la délimitation des Etats, notamment en ce qui touche l'efficacité des moyens de coercition. 